\title{
Responsabilidad social: un análisis de la situación actual en México y España ${ }^{1}$
}

\author{
Nélida Porto Serantes* \\ Juan Luis Castromán Diz**
}

\begin{abstract}
Resumen
En este trabajo nos acercamos en primera instancia al concepto de responsabilidad social, el cual ha sido objeto reciente de múltiples iniciativas emanadas de diversas organizaciones, algunas de carácter mundial; posteriormente, examinamos qué se entiende por responsabilidad social en México y España y cuál es la situación actual de su implantación en ambos países; para averiguar esto último empleamos dos vías complementarias: 1) a través de organizaciones de ámbito nacional, las cuales se centran en el uso del distintivo ESR de CEMEFI, en México, y la certificación SGE 21 de Forética, en España; y 2) según las adhesiones al Global Compact de la ONU, que consiste en evaluar las adhesiones al Pacto Mundial o Global Compact de la ONU en ambos países.
\end{abstract}

Debido a que el concepto de responsabilidad social se ha introducido recientemente en México, la información de este trabajo se obtuvo directamente del Global Compact, así como de empresas en proceso de adhesión.

Nuestro objetivo final es averiguar la acogida que ha tenido el término responsabilidad social en ambos países, así como su reciente evolución.

Palabras clave: responsabilidad social (RS), empresa socialmente responsable (ESR), sistema de evaluación ética y socialmente responsable (SGE 21), Global Compact.

\footnotetext{
1 Una versión preliminar de este artículo fue presentada en la XIV Internacional Conference of AEDEM, celebrado en Morelia en septiembre de 2005.

* Profesora investigadora de la Universidad de Santiago de Compostela, España. Correo electrónico: efnelida@usc.es

**Profesor investigador de la Universidad de Santiago de Compostela, España. Correo electrónico: oejlcd@usc.es
} 
Nélida Porto Serantes Juan Luis Castromán Diz

\section{Estado de la cuestión: el concepto de responsabilidad social}

Los términos responsabilidad social empresarial o responsabilidad social corporativa (o cualquier nomenclatura utilizada) son conceptos habitualmente utilizados para referirse a la responsabilidad social (RS), el cual se suele entender como un compromiso voluntario asumido por las organizaciones para un desarrollo sostenible.

Bestratén y Pujol (2004) lo definen de la siguiente manera:

"[...] la responsabilidad social (en adelante RS) no es algo novedoso en el mundo empresarial, aunque en estos últimos años está adquiriendo una nueva dimensión por el especial interés que conlleva, demandando una gestión cuidada como en cualquier ámbito de valor estratégico, que supere lo anecdótico o lo filantrópico".

Existen varios intentos de dar respuesta a lo que debe ser la RS; los más recientes y significativos surgen por instancias de organismos internacionales; Bestratén y Pujol (2004) retoman estos criterios en una tabla (que presentamos más adelante), en la cual observamos que, pese a las fechas ahí señaladas, algunos de estos organismos vienen realizando esta tarea desde hace tiempo. Así, por citar sólo algunos ejemplos: la Organización Internacional del Trabajo (OIT) viene desarrollando desde 1919 un sistema de normas internacionales con el fin de que hombres y mujeres consigan trabajos decentes y productivos en condiciones de libertad, igualdad, seguridad y dignidad; las Líneas directrices para empresas multinacionales de la Organización para la Cooperación y Desarrollo Económico (OCDE) tienen su origen en 1976 con la Declaración sobre Inversión Internacional y Empresas Multinacionales, la cual tras sucesivas modificaciones logra incorporar las directrices tal como hoy las conocemos; la primera versión de la SA8000 es de 1997. 
Responsabilidad social: un análisis de la situación actual en México y España

Tabla 1

\section{Referencias internacionales más recientes}

Modelo Europeo de Excelencia Empresarial, EFQM (2000)
UN / "Global Compact Iniciative" (2000)
OIT/Principios de actuación de empresas multinacionales (1997/2000) y conjunto
de Convenios y Recomendaciones
OCDE / Directrices para empresas multinacionales (2000)
Libro Verde de la Comisión Europea (2001)
OIT-ANDI / Manual de Balance Social (2001)
Social Accountability 8000 (2001, Acción social)
Global Reporting Iniciative 2003 (Sostenibilidad)

Fuente: Bestratén y Pujol. (2004)

Asimismo, en la tabla anterior sólo tenemos una pequeña parte de las referencias en materia de RS; como señala el World Bank (2003), son casi un millar de iniciativas que se han realizado sobre RS; por sus divergencias y diversidad la Asociación Española de Contabilidad y Administración de empresas (AECA, 2004:12) concluye que se carece de criterios comunes y generalmente aceptados en cuanto al concepto, los objetivos y los elementos de la RS.

En congruencia con estas afirmaciones, Hohnen (2005) señala que el estudioso de la RS se encuentra con que ésta puede significar y significa diferentes cosas para diversas personas a pesar de los esfuerzos que se están llevando a cabo en este sentido; además, tantas iniciativas "[...] están generando una cierta confusión sobre lo que realmente es la responsabilidad social empresarial y lo que la sociedad espera de las empresas" (De la Cuesta, 2004: 55).

Esta situación se puede resumir señalando que en la actualidad aún no se ha logrado una definición de la RS comúnmente aceptada, ni existe ninguna institución internacional con el poder de desarrollarla ${ }^{2}$. Sin embargo, paulatinamente se va acotando este concepto y, en consecuencia, se va resaltando su carácter de iniciativas voluntarias en torno a tres aspectos claves: el medioambiental, el laboral y la

\footnotetext{
2 Aunque es de destacar que en septiembre de 2004 la International Organization for Standarization (ISO) decidió iniciar un proceso de elaboración de una nueva norma que establecerá parámetros para la Responsabilidad Social Empresarial: la ISO 26000. La primera reunión formal del comité mundial de la ISO 26000 sobre Responsabilidad se celebró en Salvador de Bahía (Brasil) en marzo de 2005, dando comienzo a los trabajos preliminares para el desarrollo de la futura guía ISO 26000 sobre responsabilidad social, no certificable, cuya publicación se estima para el año de 2008.
} 
ética del comportamiento empresarial con los diversos grupos con los que se relaciona. Así, por ejemplo, en el contexto internacional, la Organización Internacional de Empleadores (OIE) — a la que pertenecen 139 federaciones nacionales de empleadores de 134 países - asume la siguiente definición sobre la RS (OIE, 2003:2): "[...] iniciativas desarrolladas de manera voluntaria por las empresas, en las que se integran inquietudes tanto sociales como medioambientales para la gestión de sus operaciones y la interacción con sus allegados".

De manera semejante, en el ámbito europeo, el Libro Verde de la UE (2001) entiende por $R S$

"[...] la integración voluntaria, por parte de las empresas, de las preocupaciones sociales y medioambientales en sus operaciones comerciales y las relaciones con sus interlocutores. Ser socialmente responsable no significa solamente cumplir plenamente las obligaciones jurídicas, sino también ir más allá de su cumplimiento invirtiendo más en el capital humano, el entorno y las relaciones con los interlocutores".

Posteriormente, examinaremos otras definiciones propuestas para la RS, particularmente en México y en España; todas ellas en un marco de valoración positiva con respecto a su desarrollo, sin embargo, no debemos ser ajenos a la visión de autores críticos que enuncian afirmaciones como las siguientes:

- Para muchas empresas el respeto de los derechos humanos, la protección del medio ambiente y la satisfacción de las necesidades básicas están en un segundo plano con respecto a las ganancias (Heydenreich, 2002).

- Muchos directivos y empresarios aún están inmersos en una cultura en la que "cualquier cosa vale mientras produzca dinero" (Hatcher, 2003).

- La RS es sólo el tributo que el capitalismo paga a la virtud, una industria por si misma, y una profesión que prospera (Crook, 2005:3).

Además, aunque las empresas se comprometan con estos conjuntos de valores mínimos, sin el marco de control adecuado, el compromiso puede quedarse en mera apariencia, lo que lleva a Fussler et al. (2004, p. 221) a indicar que "un enfoque voluntario pierde toda integridad y credibilidad si no puede demostrar su progreso". 
Responsabilidad social: un análisis de la

situación actual en México y España

En este trabajo se examinarán las adhesiones a los valores que representan la responsabilidad social, exclusivamente a través de la certificación por terceros (CEMEFI para las empresas mexicanas y FORÉTICA para las españolas) y de las adhesiones al Global Compact. Sin embargo, la vía de las certificaciones y adhesiones persigue garantizar que la información que se suministra no está sesgada, por lo que se presentan únicamente los aspectos positivos y se ocultan los menos favorables, pero a menudo es insuficiente para acreditar que la empresa ha adoptado de verdad los principios de responsabilidad social y que éstos han impregnado sus diferentes niveles organizativos, aunque en ocasiones la certificación se convierte en un fin en sí mismo. Así, es patente que algunas organizaciones buscan las certificaciones meramente con un fin publicitario y de imagen pública, por ello sólo se limitan a cumplir los aspectos formales requeridos para superar la auditoría y obtener la correspondiente certificación ${ }^{3}$. A este respecto, Castromán y Porto (2005: 99) —recordando lo sucedido en los escándalos financieros recientes (pese a la existencia de controles financieros de diverso tipo, de normativa legal y de auditorías obligatorias) - "[...] ponen el punto de mira en la necesidad de un adecuado marco de control interno que impida que el fraude y la administración inmoral hagan del compromiso con la responsabilidad social un mero intento de aparentar". Dichos autores —ras recordar que los cuatro factores ambientales facilitadores del fraude y del comportamiento organizativo inmoral son: motivación de los empleados, oportunidad percibida, bajo nivel ético de los empleados y comportamiento permisivo o inmoral de la gerencia- señalaban que "un comportamiento moral en la organización se potencia con el establecimiento de sistemas de control adecuados a las nuevas demandas sociales" y concluyen que el marco integrado propuesto por el renovado informe COSO (cuyos ocho elementos son: ambiente interno, establecimiento de objetivos, identificación de eventos, evaluación de riesgos, respuesta al riesgo, actividades de control, información y comunicación eficaz y supervisión de la totalidad de la gestión de riesgos corporativos) "[...] es enteramente consecuente con los requerimientos y valores propugnados por los códigos voluntarios de responsabilidad social corporativa, en el contexto de un desarrollo sostenible" (Castromán y Porto, 2005:99).

\footnotetext{
Esto, unido al hecho de que son las empresas las que eligen a las compañías auditoras a las que pagan por sus servicios, lleva a Boiral (2003A:335) a afirmar que "asegurar que una empresa determinada realmente aplica el conjunto de normas y procedimientos por el hecho de haber sido auditada es muy aventurado",de forma análoga a lo que ocurre con las auditorías referidas a la ISO 9000 y a la ISO 14000 de las que este autor demuestra en un estudio (Boiral, 2003B:773) el "relativamente modesto apoyo que merece la ISO 9000 entre el personal de las organizaciones certificadas", y esto pese a que, "[...] debido en gran parte al ritual superficial con que se lleva a cabo la auditoría, el sistema de calidad aparece coherente y ordenado, ocultando las divergencias y hasta las disidencias que pueden existir en su seno".
} 
A pesar de lo anterior, es importante destacar que en el estudio llevado a cabo por la Economist Intelligence Unit (2005), se preguntó sobre la importancia de la responsabilidad social corporativa (RSC) a un total de 136 directivos de compañías de varios países y 65 inversores. El resultado obtenido fue que el 85\% señalan a RSC como una consideración "central" o "importante" en sus decisiones en materia de inversión; lo más significativo es la evidencia del aumento de la importancia de la RSC, que casi se duplicó en un lustro, pues llegó a esos resultados partiendo de un índice del $44 \%$ en la misma encuesta realizada cinco años antes.

En este sentido, Werther y Chandler (2005:317) señalan:

[...] mientras nos adentramos más en el siglo XXI, afirmamos que la maximización de beneficio y la RSC llegarán a ser cada vez más inseparables. En última instancia, la RSC afecta a ambos, a la legitimación social de la empresa y a la percepción de los grupos de interés, que alternadamente afectan al funcionamiento económico, especialmente para los negocios basados en la marca de fábrica.

Orlitzky (2005), en su primer principio, postuló que la gestión de la responsabilidad social/medioambiental es un elemento legítimo y cada vez más importante de la estrategia de negocio.

\section{Responsabilidad social corporativa en México}

La diversidad de definiciones y aproximaciones a la responsabilidad social también se constata en el ámbito mexicano, como lo indica Gómez (2003:8):

[...] no se cuenta con una percepción única y homogénea de lo que es la responsabilidad social empresarial (RSE) por lo que las prácticas que se realizan en su nombre son diversas algunas concepciones son cercanas a la caridad o donaciones de dinero, tiempo y talento hacia obras sociales; otros entienden la RSE como una estrategia hacia dentro de la empresa que permita garantizar mejores condiciones laborales a sus trabajadores; en otros casos se percibe como impulsos de mejora hacia la comunidad en donde se localizan las empresas, bajo una lógica de adoptar valores éticos; también se le ubica como una estrategia dirigida hacia los stakeholders bajo un enfoque de "ganar-ganar" y otros la perciben como una forma de asumir la dirección estratégica de la empresa de tal forma que se constituya un planteamiento integral para el manejo organizacional como una acción estratégica y de largo plazo articulada a la dinámica interna de la empresa y su entorno. 
Responsabilidad social: un análisis de la situación actual en México y España

Diferentes asociaciones civiles y organismos no gubernamentales en México promueven y apoyan la responsabilidad social corporativa. Pionero es el Centro Mexicano para la Filantropía"4 (CEMEFI) para el que la empresa socialmente responsable (ESR) es:

[...] aquella que tiene el compromiso consciente y congruente de cumplir integramente con la finalidad de la empresa tanto en lo interno y lo externo, considerando las expectativas de todos sus participantes en lo económico, social o humano y ambiental, demostrando el respeto por los valores éticos, la gente, las comunidades, el medio ambiente y para la construcción del bien común.

Por su parte el CEMEFI entiende por RS:

[...] la conciencia del compromiso y la acción de mejora continua medida y consistente, que hace posible a la empresa ser más competitiva, cumpliendo con las expectativas de todos sus participantes en particular y de la sociedad en lo general, respetando la dignidad de la persona, las comunidades en que opera y su entorno.

Este CEMEFI otorga un certificado, previa revisión anual, a las empresas que realicen acciones en cuatro ámbitos:

1) Contribuir a la calidad de vida dentro de la empresa.

2) Cuidado y preservación del medio ambiente.

3) Desempeñarse con un código de ética.

4) Vincularse con la comunidad a partir de la misión del negocio pero también desde los bienes y servicios producidos.

Además las empresas deben asumir, adoptar y publicar los indicadores ESR que señalan el grado de adopción de la responsabilidad y de su decálogo, lo cual se incluye en la tabla siguiente.

4 CEMEFI, fundada en 1988, es una institución privada con ámbito de acción circunscrito a México. Su órgano colegiado de autoridad es la Asamblea General de Asociados que está integrada por 625 miembros: 174 socios (82 fundaciones y asociaciones operativas, 39 personas y 53 empresas), 432 afiliados y 19 usuarios localizados en todo el país. A las empresas le ofrece, entre otros, los siguientes servicios:

- Apoyo en el diseño de estrategias de responsabilidad social empresarial

— Vinculación con especialistas en el diseño de programas de inversión en la comunidad.

- Identificación y difusión de las mejores prácticas de responsabilidad social empresarial. 
Nélida Porto Serantes Juan Luis Castromán Diz

Tabla 2 Decálogo de la empresa socialmente responsable de CEMEFI

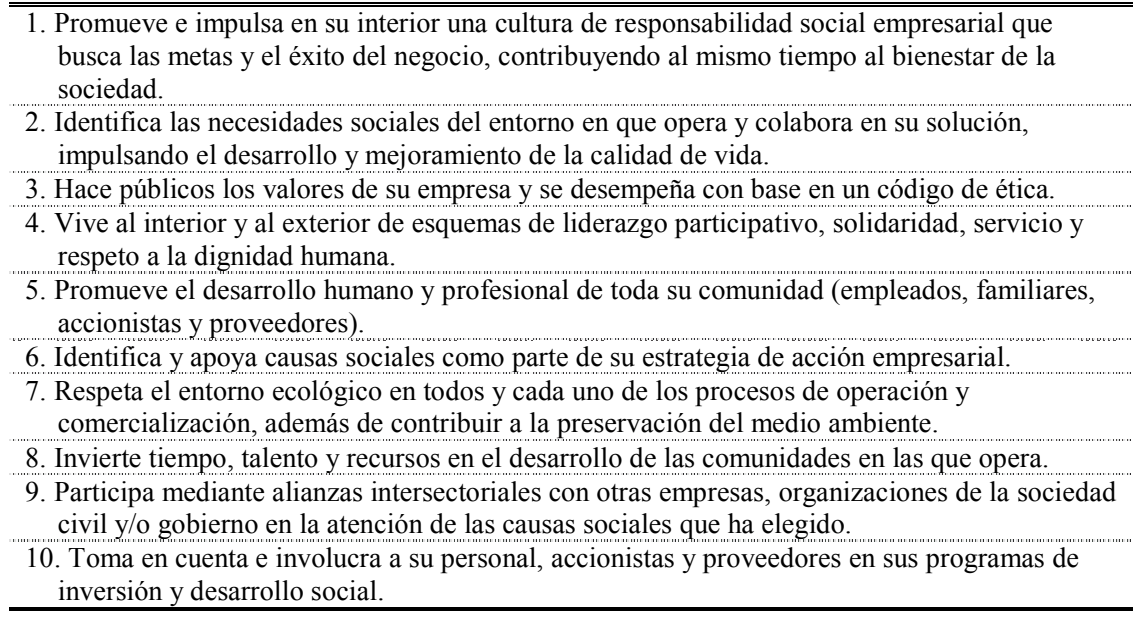

\section{Fuente: CEMEFI (2001)}

Al modelo de RS del CEMEFI para la obtención del distintivo ESR la misma CEMEFI la define como:

[...] es un proceso de confianza, honestidad y transparencia empresarial, cuyos indicadores son afines a lineamientos nacionales e internacionales, lo cual garantiza su validación frente a organismos internacionales como son entre otros: UN Global Compact Principles, Business in the Community, Caux Round Table Principles, Código de Mejores Prácticas Corporativas (CCE), Convenio de lucha contra la Corrupción (OCDE), Great Place to Work, Declaración Universal de los Derechos Humanos, Declaración de Principios Fundamentales y Derechos del Trabajo (OIT), Documento "Empresas del Nuevo Siglo"(koffi Annan), Global Sullivan Principles, Guía de Inicio (Forum Empresa), Modelo de Equidad y Género 2003 Inmujeres, SA 8000, Empresa Transparente SECODAM, Norma AA. 1000.

Desde la implantación de este certificado en el año 2001, las empresas mexicanas que han logrado el distintivo ESR han ido en aumento, tal como refleja la tabla 3; se parte de 17 que inicialmente lo obtuvieron en el año 2001 y se llega hasta las 84 que lo disfrutan durante el año 2005. 
Tabla 3

Empresas mexicanas que han obtenido el distintivo ESR (empresas socialmente responsables)
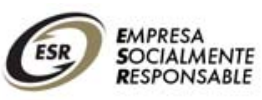

\begin{tabular}{lccccc}
\hline Año & 2001 & 2002 & 2003 & 2004 & 2005 \\
\hline \hline $\mathrm{N}^{\circ}$ de empresas & 17 & 28 & 41 & 61 & 84 \\
\hline
\end{tabular}

Fuente: http://www.cemefi.org/

Es importante señalar que de las 17 empresas que inicialmente obtuvieron el distintivo ESR en el 2001 hasta el 200512 han logrado todos los años el distintivo ESR otorgado por CEMEFI (tabla 4). Ahí mismo también se observa que son 10 las empresas que renovaron su distintivo en su cuarto año consecutivo (de las 11 empresas que podrían haberlo logrado).

Tabla 4

Empresas mexicanas que repiten en la obtención del distintivo ESR

\begin{tabular}{|c|c|c|c|}
\hline $\begin{array}{c}\mathrm{N}^{0} \text { de años a acumulados que } \\
\text { obtuvieron la distinción }\end{array}$ & 2004 & 2005 & $\begin{array}{c}\mathrm{N}^{\circ} \text { de años a acumulados que } \\
\text { obtuvieron la distinción }\end{array}$ \\
\hline$\overline{T 1}$ & 21 & 27 & $\bar{~} \overline{1}$ \\
\hline 2 & 17 & 20 & 2 \\
\hline 3 & 11 & 15 & 3 \\
\hline \multirow[t]{3}{*}{$4(2001-2004)$} & 12 & 10 & 4 \\
\hline & & 12 & $5(2001-2005)$ \\
\hline & 61 & 84 & \\
\hline
\end{tabular}

Elaborado a partir de los datos de http://www.cemefi.org/

Por último, incluimos en la tabla siguiente el listado de las empresas socialmente responsables para CEMEFI en el 2005; es decir, las ochenta y cuatro organizaciones que ostentan el distintivo ESR durante ese año. 
Tabla 5

84 empresas mexicanas socialmente responsables según CEMEFI

\begin{tabular}{|c|c|c|}
\hline 1 & Accor & \begin{tabular}{l|l|}
43 & Hipotecaria SuCasita \\
\end{tabular} \\
\hline 2 & Administración Portuaria Integral de Coatzacolacos & 44 Holcim Apasco ESR desde 2001 \\
\hline 3 & ADO y Empresas Coordinadas (Región Caribe) & 45 Industrias Peñoles, \\
\hline 4 & Agrícola San Isidro de Culiacán & 46 Inmobiliaria Parque Reforma \\
\hline 5 & Agrícola Tarriba S. de R. L. de C. V. (ahora Ceuta Produce) & 47 Interceram ic \\
\hline 6 & Agro Industrias del Norte & 48 Interprotección, Agente de Seguros y Fianzas \\
\hline 7 & AM ANCO México & 49 J.P. Morgan Grupo Financiero \\
\hline 8 & American Express Company (México) & 50 Janssen Cilag México \\
\hline 9 & Arancia Industrial & 51 Manpower \\
\hline 10 & Auto A horro Automotriz (Planfía) & 52 Mc Donald's de México \\
\hline 11 & Avantel & 53 Merck Sharp \& Dohme de México \\
\hline 12 & Boehringer Ingelheim Promeco & 54 Metlife México \\
\hline 13 & CEMEX & $55 \mid$ Microsoft de México \\
\hline 14 & Cinépolis & 56 Mittal Steel Lázaro Cárdenas \\
\hline 15 & Coca Cola FEMSA & 57 Monex Grupo Financiero \\
\hline 16 & Comisión Federal de Electricidad Centro Sur & 58 Nadro \\
\hline 17 & Comisión para la Regularización para la Tenencia de la Tierra (CORETT) & 59 Nes tlé de México \\
\hline 18 & Compañía Mexicana de Aviación & 60 Nextel de México \\
\hline 19 & Cooperativa La Cruz Azul & 61 Novartis Farmaceútica \\
\hline 20 & Danone de México & 62 Office Coffee Service \\
\hline 21 & Deloitte (antes Galaz, Yamasaki, Ruiz Urquiza / Deloitte Consulting) & 63 OXXO Femsa Comercio \\
\hline 22 & Desarrolladora Homex & 64 Parque Garrafón \\
\hline 23 & Diconsa & \begin{tabular}{l|l}
65 & Parque X caret
\end{tabular} \\
\hline 24 & Embotelladoras Arca & \begin{tabular}{l|l|}
66 & Parque Xel-Há \\
\end{tabular} \\
\hline 25 & FEMSA Insumos E stratégicos & $67 \mid$ Pfizer \\
\hline 26 & Financiera Independencia & 68 Philips Mexicana \\
\hline 27 & FinComún, Servicios Financieros Comunitarios & 69 Promoción y Operación (PROSA) \\
\hline 28 & Fondo Nacional para el Fomento de las Artesanías (Fonart) & $\begin{array}{ll}70 & \text { S.C. Johns on and Son ESR desde } 2001\end{array}$ \\
\hline 29 & Ford Motor Company & 71 Seguros Monterrey New York Life \\
\hline 30 & GE (General Electric) & 72 Shell México ESR desde 2001 \\
\hline 31 & Grupo Ángeles Servicios de Salud ESR des de 2001 & 73 Sodexho México \\
\hline 32 & Grupo Bimbo & 74 Supermercados Internacionales HEB $\quad$ ESR desde 2001 \\
\hline 33 & Grupo Financiero BBVA Bancomer ESR desde 2001 & 75 Tecnológico de Monterrey (Campus Cuernavaca) \\
\hline 34 & Grupo Financiero Santander Serfin & 76 Tecnológico de Monterrey (Campus Edo. de México) \\
\hline 35 & Grupo Gamesa & 77 Tecnológico de Monterrey IT ESM (Campus Monterrey) \\
\hline 36 & Grupo Industrial Lala & 78 Telefónica Móviles México \\
\hline 37 & Grupo Jugos Del Valle & 79 The Coca Cola Export Corporation México ESR desde 2001 \\
\hline 38 & Grupo Modelo Empresas de Servicios & 80 Universal Productora \\
\hline 39 & Grupo Nacional Provincial & 81 Vía Delphi \\
\hline 40 & Grupo Telmex & 82 Wal-Mart de México \\
\hline 41 & Hewlett Packard México $\quad$ ESR desde 2001 & 83 Wyeth \\
\hline 42 & Hipotecaria Crédito y Casa & 84 Zimat Consultores ESR desde 2001 \\
\hline
\end{tabular}

\section{Responsabilidad social corporativa en España}

En el ámbito español, en el marco de la Unión Europea (UE), la Asociación Española de Contabilidad y Administración de Empresas (AECA, 2004:21) define la RSC de la siguiente forma:

[...] el compromiso voluntario de las empresas con el desarrollo de la sociedad y la preservación del medio ambiente, desde su composición social y un comportamiento responsable hacia las personas y grupos sociales con quienes se interactúa [que] centra su atención en la satisfacción de las necesidades de los 
Responsabilidad social: un análisis de la situación actual en México y España

grupos de interés [y que] va más allá del mero cumplimiento de la normativa legal establecida y de la obtención de resultados exclusivamente económicos a corto plazo.

Los elementos conceptuales que AECA (2004:20) identifica en la responsabilidad social corporativa (RSC) son:

- Responsabilidad: capacidad para reconocer, aceptar y, en su caso, plantear respuestas a las consecuencias de un comportamiento realizado consciente y libremente

- Social: cualidad de la persona como elemento integrante de un sistema social o colectivo de individuos.

- Corporación: entidad de interés público, comúnmente asociada a un proyecto económico.

- Compromiso voluntario: obligación contraída por voluntad propia.

- Organización: conjunto de personas estable, institucionalizado y estructurado adecuadamente, dotado de medios para alcanzar unos fines.

- Sociedad: agrupación de personas, que constituyen unidad distinta de cada uno de sus individuos con el fin de cumplir, mediante mutua cooperación, todos o alguno de los fines de la vida.

- Medio ambiente: entorno físico natural, incluidos el aire, agua, tierra, flora, fauna y recursos no renovables.

- Persona: individuo, humano.

- Grupos de interés: partes interesadas, o stakeholders, afectados de una u otra manera por la existencia o acción de las organizaciones y con algún interés sobre ellas.

A diferencia de lo que ocurre en México con CEMEFI, AECA no propone un modelo propio, sino que apoya explícitamente la emisión de información sobre responsabilidad social, utilizando el modelo propuesto por la Global Reporting Initiative $(\mathrm{GRI})^{5}$.

\footnotetext{
5 En el marco del Global Compact nace la GRI (Global Reporting Initiative) una guía de buenas prácticas de información de las organizaciones en el ámbito internacional. Como indica Porto (2005:30), el modelo GRI impulsa la armonización internacional en la divulgación de información relevante y creíble en tres ámbitos: medioambiental, económico y social, a través de la elaboración de una Memoria de Sostenibilidad. La versión actual es la del año 2002, la cual se halla en proceso de revisión, que culminará en octubre del año 2006. Con el fin de certificar la veracidad de la información suministrada, para proporcionarle la credibilidad que la sociedad demanda, se apoya en la ACCOUNTABILITY 1000 (AA 1000), una norma desarrollada por ISEA (Institute of Social and Ethical Accountability) en 1999.
} 
Por otra parte, el gobierno de España, para dar un impulso al desarrollo de la RS, creó el Foro de Expertos de RSE, cuyo objetivo durante este año, en palabras del ministro Jesús Caldera (2005) "[...] es que estudie, debata y proponga medidas para fomentar las prácticas de ética social y medioambiental, dentro del marco del desarrollo sostenible definido por las instituciones de la Unión Europea".

Fruto del trabajo de este Foro, compuesto por representantes de distintos ministerios y expertos provenientes de organizaciones de la sociedad civil y de la Universidad, es su concepción de la responsabilidad social de la empresa (RSE) como aquella que tiene por objetivo "[...] la sostenibilidad basándose en un proceso estratégico e integrador en el que se vean identificados los diferentes agentes de la sociedad afectados por las actividades de la empresa". Además, se añade que "para su desarrollo deben establecerse los cauces necesarios para llegar a identificar fielmente a los diferentes grupos de interés y sus necesidades, desde una perspectiva global y se deben introducir criterios de responsabilidad en la gestión que afecten a toda la organización y a toda su cadena de valor" (2005:3).

En 2000 se creó en España una asociación sin ánimo de lucro denominada FORÉTICA $^{6}$ para que fuera un foro multidisciplinar de la responsabilidad social. Uno de sus primeros frutos fue la elaboración de la Norma de Empresa SGE 21, en la que participaron más de 100 expertos representando a todos los grupos de interés. Esta norma, lanzada en 2002, recoge el primer sistema de gestión de la responsabilidad social europeo que permite, de manera voluntaria, alcanzar una certificación. Urtiaga y Granda (2001) consideran la SGE 21 es un sistema objetivo de implantación, evaluación y verificación de la gestión ética en las organizaciones.

6 FORÉTICA es una asociación sin ánimo de lucro que surge en el seno del X Congreso de Empresas de Calidad, de Barcelona, 1999, cuando un grupo de directivos y líderes de opinión se plantea qué hacer para crear una cultura de empresa basada en la responsabilidad de todos sus miembros frente al conjunto de la sociedad y el entorno.

La actividad de FORÉTICA persigue dos objetivos principales:

- Ser el referente en servicios de valor ligados a la responsabilidad social de las organizaciones.

- Ser un gestor de conocimiento. 
Responsabilidad social: un análisis de la situación actual en México y España

La norma SGE 21 conlleva la aplicación de procedimientos comunes para la auditoría y la aceptación del resto de las reglas del juego que desarrolla FORÉTICA. Su cumplimiento permite el disfrute de la marca "miembro de FORÉTICA", que se exterioriza con el uso de un logotipo (véase tabla 6) ${ }^{7}$. Para Urtiaga y Granda (2003) "[... ] llegar a certificar la Gestión de la Organización de acuerdo con esta norma, de empresa, supone adquirir voluntariamente un Compromiso Social para aplicar los valores éticos incorporados en la misma, demostrarlo y mantenerlo".

El SGE 21 es válido por tres años, además es revisado con auditorías anuales de seguimiento en las organizaciones para comprobar el mantenimiento y la mejora de las condiciones observadas en la auditoría inicial, a continuación se presentan las empresas certificadas:

Tabla 6

Compañías certificadas en SGE 21 de FORÉTICA

\begin{tabular}{|c|c|c|}
\hline \multirow{5}{*}{ Forética } & 1 & NOVARTIS FARMACEUTICA \\
\hline & 2 & CIBA VISIÓN \\
\hline & 3 & CONTRATAS Y OBRAS \\
\hline & 4 & EUROFIRMS ETT \\
\hline & 5 & CONSTRUCCIONES LLABRÉS FELIÚ \\
\hline \multirow{7}{*}{$\frac{\text { Gestión Ética }}{\text { GE } 02 / 03}$} & 6 & RED POSTAL TRANSFER DE CORREOS Y TELÉGRAFOS \\
\hline & 7 & FROSST IBÉRICA (GRUPO MSD) \\
\hline & 8 & CÁMARA DE COMERCIO E INDUSTRIA DE ALAVA \\
\hline & 9 & FUNDACIÓN SAN JOSÉ ETXE-ALAI \\
\hline & 10 & URBAGASA NUEVE, S.L. \\
\hline & 11 & TECNOESPACIO PROYECTOS, S.L. \\
\hline & 12 & VISTAZUL PROYECTOS, S.L. \\
\hline & 13 & GEROGESTIÓN, SERVICIOS GERONTOLÓGICOS, S.A. \\
\hline & 14 & CONSTRUCCIONS DECO \\
\hline & 15 & VERTISUB \\
\hline
\end{tabular}

\section{Fuente FORÉTICA, http://www.foretica.es, consultada el 14 de octubre de 2005}

\footnotetext{
7 La norma SGE 21 de FORÉTICA cumple con los requisitos del Pacto Mundial de las Naciones Unidas, de la Declaración tripartita de la OIT sobre las empresas multinacionales y la política social, de las Directrices de la OCDE para las empresas multinacionales, de la Comunicación de la Comisión relativa a la responsabilidad social de las empresas: una contribución empresarial al desarrollo sostenible del 2002 de la Social Accountability 8000 y de la UNE-EN-ISO 9000:2000 y normas relacionadas (FORÉTICA, 2002:6).
} 
Para la SGE 21 la organización se subdivide en ocho áreas de gestión (alta dirección, relaciones con clientes, relaciones con proveedores, relaciones con personal propio, con el entorno social, con el accionariado, con la competencia y con las administraciones competentes) y determina para cada una de ellas una serie de valores para aplicar tales como honradez y lealtad hacia los clientes, reconocimiento de derechos a los trabajadores y asunción de responsabilidades con respecto a los mismos, etcétera.

Las empresas que han conseguido la certificación SGE 21 de FORÉTICA (tabla 6) elaboran un conjunto de documentos interrelacionados y una serie de Registros, que permiten a la organización demostrar tanto internamente, mediante una auditoría interna, como externamente, a través de una auditoría por tercera parte, que su actuación es consecuente con el compromiso adquirido de implantar un sistema de gestión ética.

\section{Implantación del Pacto Mundial en México y España}

La otra vía que proponemos para analizar la situación de la RS en ambos países es la implantación del Pacto Mundial (Global Compact) de la ONU para México y España.

Debemos destacar que el Pacto Mundial de las Naciones Unidas, tal como indica en su propia web:

[...] es una iniciativa de compromiso ético destinada a que las entidades de todos los países acojan como una parte integral de su estrategia y de sus operaciones, diez Principios de conducta y acción en materia de Derechos Humanos, Trabajo, Medio Ambiente y Lucha contra la Corrupción.

La adhesión al Global Compact es una de las vías reconocidas internacionalmente para que las empresas demuestren su compromiso con la RS, cuyos apoyos (tanto en España y México como en el resto del mundo) han ido creciendo hasta abarcar a 2.328 empresas, tal y como se muestra en la tabla siguiente. 
Responsabilidad social: un análisis de la situación actual en México y España

Tabla 7

Adhesiones de empresas al Pacto Mundial de la ONU

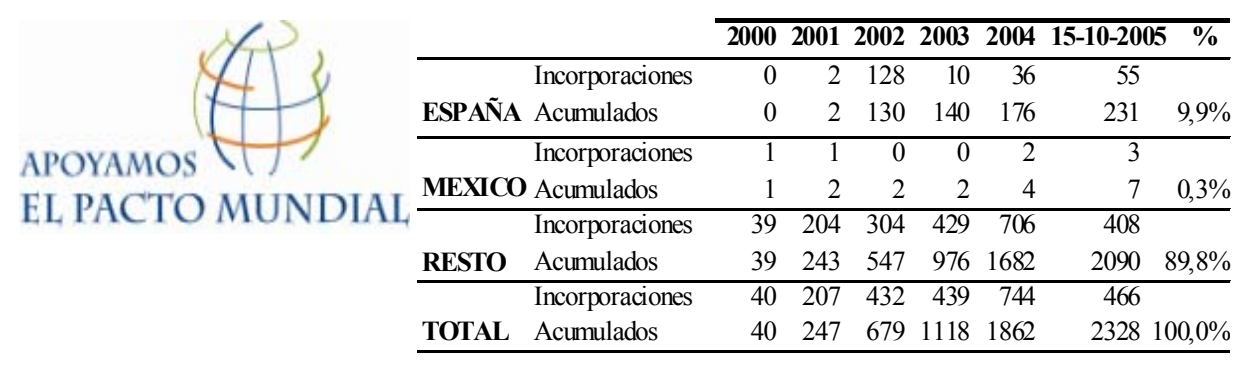

\section{Elaborado a partir de los datos del Global Compact, consultados el 15/10/05 http://www.unglobalcompact.org/}

En esta tabla podemos observar que España presenta en la fecha de elaboración de este trabajo 231 empresas que se han adherido al Pacto Mundial y México 7, según los datos de la página oficial del Global Compact de Naciones Unidas. Una cuestión por tomar en cuenta es que Pacto Mundial fue presentado el 9 de junio de 2005 en México, lo que explica su escasa implantación, en cambio en España, la implantación es mayor por haber sido presentado el 9 de abril de 2002.

Sin embargo, conviene considerar que la tabla está elaborada sobre los datos suministrados por el propio Global Compactal a 15 de octubre de 2005 y que en el caso de México (véase al tabla 8) el Centro Mexicano para la Filantropía (CEMEFI) reporta que hay 32 empresas mexicanas vinculadas a él (al 7 de junio de 2005), adheridas o que están en proceso de adhesión al Pacto Mundial, especificando además que algunas empresas que operan en México pueden estar adheridas a través de sus matrices; por otra parte, también puntualiza que ha detectado a la empresa socialmente respetable (ESR) que cumple con los principios del Pacto Mundial aunque no han decidido suscribirlo. 
Tabla 8

Empresas mexicanas vinculadas al CEMEFI adheridas o en proceso de adhesión al Pacto Mundial* (al 7 de junio de 2005)

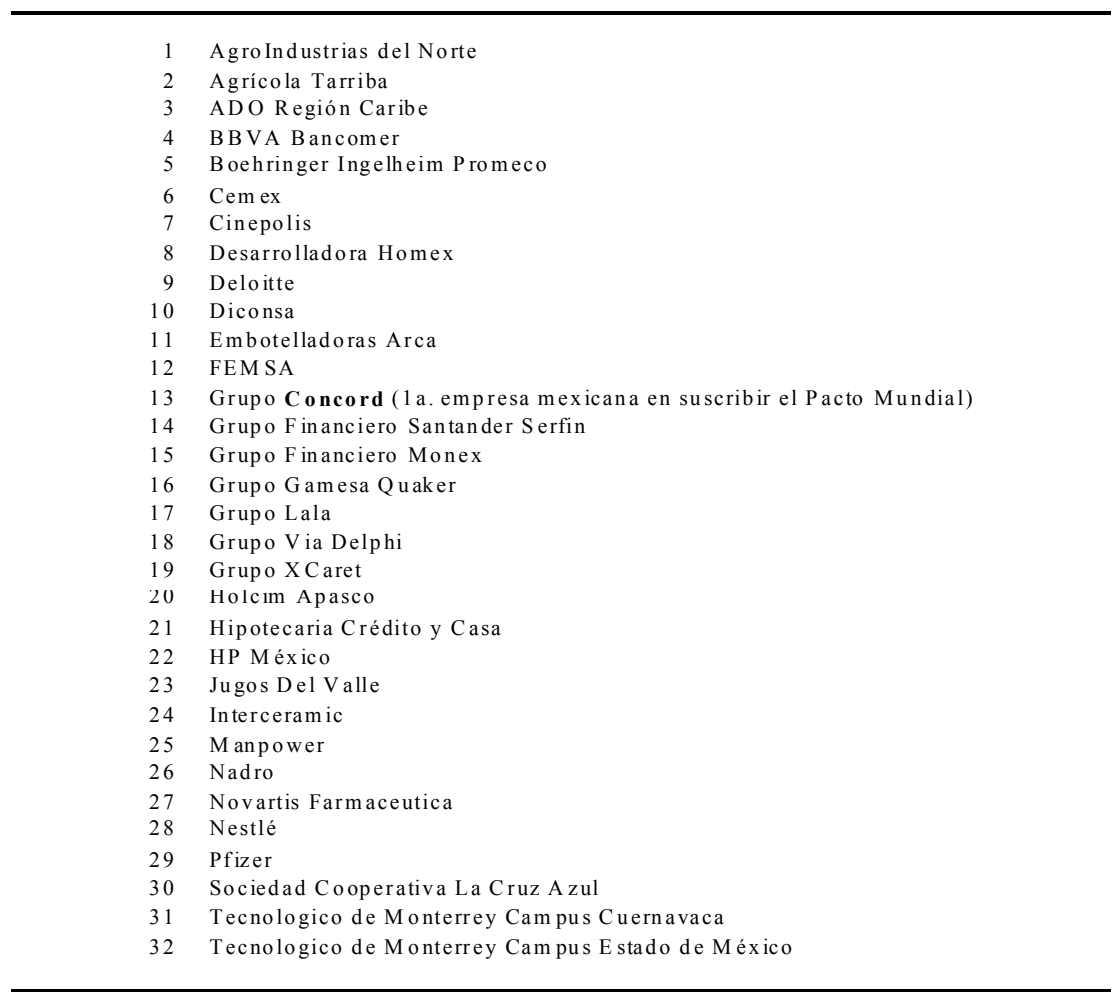

\section{Fuente: CEMEFI \\ (http://www.cemefi.org/index.cfm?page=CEM_RSE_22\#c)}

Después de consultar la página Web de COMPITE $^{8}$ el 15 de octubre de 2005, observamos que allí afirman que son casi 250 las organizaciones que están adheridas al Pacto Mundial; estos datos en todo caso anuncian la importante aceptación que va ganando esta iniciativa en México pese a su reciente presentación en

8 El Comité Nacional de Productividad e Innovación Tecnológica, A.C. (COMPITE) fue fundado en 1997. Es una asociación civil sin fines de lucro cuyo objeto social es promover la productividad e inducir procesos de calidad y de responsabilidad social en las micro, pequeñas y medianas empresas a través de la incorporación de nuevas tecnologías para mejorar su competitividad (http://:www.compite.org.mex) 
el país. Asimismo, en la página del Pacto Mundial-España encontramos que hasta el 15 de octubre de 2005 se indica que son 277 las empresas que están adheridas al Pacto. Este número es claramente superior en 46 empresas al reflejado en la página del Global Compact. Acudimos también a la web del Pacto Mundial en México para contrastar las cifras, y en esa misma fecha la base de datos aún se hallaba en fase de construcción. Aún así, con las salvedades que las cifras pudieran aconsejar es incuestionable la importancia que está adquiriendo esta iniciativa de la ONU.

Por otra parte, es importante señalar que uno de los incentivos para adherirse al Pacto Mundial es poder utilizar su logo (incluido en la tabla 7), el cual lo pueden utilizar las organizaciones adheridas al Pacto Mundial al lado del suyo propio, tanto como elemento permanente de su papelería, tarjetas de presentación o cualquier otra variante de material impreso como para promover o anunciar productos y servicios del participante en el Pacto Mundial siempre que no sea usado como una certificación de origen.

Ahora bien, entendemos que los distintivos que pueden utilizar las organizaciones no son suficientes para incentivar a las empresas a ser más responsables social y medioambientalmente. En este sentido, coincidimos con Lizcano y Moneva (2004): "[...] el conocimiento y el grado de implantación de la RSC va a depender directamente de la divulgación y reconocimiento público otorgados a las mejores prácticas".

Con esta intención se ha creado en España el premio a la Mejor Información de Sostenibilidad, organizado por el Instituto de Censores Jurados de Cuentas de España (ICJCE) y la Asociación Española de Contabilidad y Administración de Empresas (AECA), que es clasificatorio para los X Premios ESRA (European Sustainability Reporting Awards).

\section{Conclusiones}

Después de revisar el concepto de responsabilidad social, tanto en el ámbito internacional como en México y España para averiguar la situación actual de su implantación en ambos países podemos concluir que: 
1) Aún no se ha logrado una definición de responsabilidad social comúnmente aceptada, ni existe un consenso en cuanto a sus objetivos y elementos. En España y México se entiende que la responsabilidad social se relaciona con la adhesión a las diferentes iniciativas de forma voluntaria en los ámbitos medioambiental, social y ético, corroborando la importancia, cada vez mayor, que está adquiriendo la responsabilidad social en el mundo empresarial.

2) Tanto en México como en España, existen distintivos otorgadas por instituciones de carácter privado que certifican la implantación de la RS. En México, la iniciativa de CEMEFI promueve el distintivo de empresa socialmente responsable (ESR), que han logrado un total de 84 empresas en 2005, mientras que en España existen 13 organizaciones que han conseguido el distintivo SGE 21 de FORÉTICA en el mismo año; por lo tanto, es una vía de mayor aceptación en México.

3) Si atendemos a las adhesiones a una iniciativa internacional, como es el Pacto Mundial de la ONU, y según los datos suministrados por el propio Global Compact, México con 7 empresas adheridas presenta un número inferior al de España, que agrupa a 231 empresas adheridas. Entendemos que esta diferencia se debe a que el Pacto Mundial fue presentado en México en 2005, mientras que en España fue lanzado en 2002; en todo caso, se constata la rápida implantación que está logrando en ambos países.

4) Además, si complementamos la información obtenida directamente del Global Compact con otra referida a empresas en proceso de adhesión — suministrada por instituciones como CEMEFI que señala que son 84 las empresas adheridas o en proceso de adhesión al Pacto Mundial, como COMPITE que reporta que son cerca de 250 empresas las que se hallan en este proceso - vemos que los datos para México son mucho más significativos, pese a ser presentada en sociedad esta iniciativa, hace tan solo cuatro meses. 
Responsabilidad social: un análisis de la

situación actual en México y España

5) El Pacto Mundial-España, reporta que son 46 empresas más las adheridas de las que recoge la página del Global Compact, lo que alcanzó un total de 277 empresas; en todo caso, al igual que acontece en México, se constata un creciente número de empresas españolas motivadas para ser cada vez más responsables.

6) Si nuestro objetivo final era averiguar la acogida en ambos países del concepto de responsabilidad social, así como su reciente evolución, hemos de concluir una evolución rápida y favorable a su implantación; en consecuencia existe un compromiso ético cada vez mayor de las empresas con su entorno social, medioambiental y con los grupos de allegados, a pesar de la visión critica que algunos autores intentan dar de la responsabilidad social.

\section{Bibliografía}

ASOCIACIÓN ESPAÑOLA DE CONTABILIDAD Y ADMINISTRACIÓN DE EMPRESAS (2004), Marco conceptual de la responsabilidad social corporativa, Documento AECA, Madrid.

BESTRATÉN BELLOVÍ, M. y L. Pujol Senovilla (2004), "NTP:643. Responsabilidad social de las empresas (I): conceptos generales". Instituto Nacional de Seguridad e Higiene en el trabajo, Ministerio de Trabajo y Asuntos Sociales, España.

BOIRAL, O. (2003A), "The Certification of Corporate Conduct: Issues and Prospects", International Labour Review, vol.142, no.3, Switzerland, pp. 317-340.

— (2003B), "ISO 9000, Outside the Iron Cage", Organization Science, vol. 14 , no. 6 , pp. $720-737$.

CALDERA SÁNCHEZ-CAPITÁN, J. (2005), "Notas de saludo al Foro de Expertos de RSE", 17 de marzo, en http://www.mtas.es/Empleo/economia-soc/ NoticiasDoc/NoticiasPortada/SaludoForoExpertos.htm 
CASTROMÁN DIZ, J. L. y N. Porto Serantes (2005), "Responsabilidad social y control interno". Revista Universo Contábil, Blumenau, v. 1, n. 2, maio/ ago, Brasil, pp. 86-101.

—_(1999), "La responsabilidad social de la empresa: Impacto social, comportamiento empresarial e información pública", XIII Congreso Nacional y IX Congreso Hispano Francés, AEDEM, Logroño, pp. 471-477

CEMEFI (2001); Decálogo de la Empresa Socialmente Responsable http://www.cemefi.org/index.cfm?page=CEM_RSE_39\#c

COMISIÓN DE LAS COMUNIDADES EUROPEAS COM (2001), "Fomentar un marco europeo para la responsabilidad social de las empresas", Libro verde.

Comunicación de la Comisión relativa a la responsabilidad social de las empresas: una contribución empresarial al desarrollo sostenible / COM/2002/ 0347 final.

CROOK C. (2005), "The good company", Economist, 22 de enero de 2005, vol. 374, Issue 8.410, pp.3-5.

DE LA CUESTA GONZÁLEZ, M.(2004), "El porqué de la responsabilidad social corporativa", Boletín Económico de ICE, $\mathrm{N}^{\circ} 2813$, del 2-5 de septiembre, pp. 45-58.

EUROPEAN MULTISTAKEHOLDER FORUM ON CSR (2004), Report of the Round Table on "Improving knowledge about CSR and Facilitating the Exchange of Experience and Good Practice', en http://europa.eu.int/comm/ enterprise/csr/documents/knowledge_rt_final_report.pdf.

ECONOMIST INTELLIGENCE UNIT (2005), The importance of corporate responsibility. http://www.oracle.com

FERNÁNDEZ KRANZ, D. y A. Merino Castelló (2005), "¿Existe disponibilidad a pagar por responsabilidad social corporativa? Percepción de los consumidores", Universia Business Review, nº 7, 3er. trimestre, Madrid, pp. 38-53. 
Responsabilidad social: un análisis de la

situación actual en México y España

FORÉTICA (2002), Norma SGE 21. Sistema de la Gestión Ética y Socialmente Responsable. Norma para la evaluación de la gestión Ética y Socialmente Responsable en las Organizaciones, julio 2002, FORÉTICA, Madrid.

FORO DE EXPERTOS EN RSE (2005), "I, II Y III Sesión de Trabajo de Foro de Expertos en RSE", Ministerio de Trabajo y Asuntos Sociales Dirección General de Economía Social, de Trabajo Autónomo y Fondo Social Europeo, consultado en http://psicondec.rediris.es/RSC/RSE.pdf, el 12 agosto de 2005.

GÓMEZ LEMUS, M.A. (2003), "Una visión de la responsabilidad social corporativa en México", Papeles de Ética, Economía y Dirección, nº 8.

GRANDA, G. y J. Silos (2004), Informe 2004. Responsabilidad Social de las Empresas. Situación en España. FORÉTICA, http://www.foretica.es.

HATCHER, T. (2003), "Ética para un nuevo mundo" Training \& Development Digest, $\mathrm{N}^{\mathrm{o}} 43$, noviembre.

HEYDENREICH, C (2002), "Prólogo a Globalización Responsable. Las líneas directrices de la OCDE para las empresas multinacionales. Una Discusión", Germanwatch e.V

HOHNEN, P. (2005), "Can corporate social responsibility survive 2005?", Ethical Corporation, 24 January 2005. http://www.ethicalcorp.com.

LIZCANO, J. L. y J. M. Moneva (2004), "Marco conceptual de la responsabilidad social corporativa", Revista AECA 68, Madrid.

ORGANIZACIÓN INTERNACIONAL DE EMPLEADORES (2003), La responsabilidad social de la empresa. El enfoque de la OIE. OIE, Ginebra.

ORLITZKY, M. (2005), "Social responsibility and financial performance: Trade-off or virtuous circle?", University of Auckland business Review, Autumn, pp. 37-43.

PORTO SERANTES, N. (2005), "Tendencias en la información no financiera para la gestión de la empresa socialmente responsable", Revista Iberoamericana de contabilidad de Gestión. № 5 . 
Nélida Porto Serantes Juan Luis Castromán Diz

PRICEWATERHOUSECOOPERS (2003), Responsabilidad Social Corporativa: Tendencias empresariales en España.

RIVERO LUENGO, P. (2003), "Responsabilidad social corporativa. Un nuevo modelo de Gestión y Medición para la Empresa", Revista AECA 64, Madrid, pp. 35-37.

URTIAGA DE VIVAR, A y G. Granda (2003), "Sistemas para la implantación de la gestión ética y socialmente responsable. situación actual", Papeles de Ética, Economía y Dirección, $\mathrm{n}^{\circ} 8$.

URTIAGA, A y GRANDA, F (2001), "La implantación del sistema de gestión ética SGE, de FORÉTICA como instrumento de liderazgo en las organizaciones". Papeles de Ética, Economía y Dirección, nº 6.

VILLAFANE \& ASOCIADOS (2005), Monitor Español de Reputación Corporativa (MERCO). http://www.villafane.com/reputacion/merco2005.pdf, consultado 31/05/05.

VIVES, A.y Peinado-Vara, E, Ed. (2004), Del Dicho al hecho. II Conferencia Interamericana sobre Responsabilidad Social de la Empresa, México.

WERTHER JR., W.B. y D. Chandler (2005), "Strategic corporate social responsibility as global brand insurance", Business Horizons no 48, pp. 317-324.

WORLD BANK (2003), Strengthening Implementation of Corporate Social Responsibility in Global Supply Chains, World. Bank.

Paginas web consultadas:

CEFEMI: http://www.cemefi.org/

COMPITE: http://www.compite.org.mx/

Global Compact: http://www.unglobalcompact.org

Pacto Mundial-España: http://www.pactomundial.org/

Pacto Mundial-México: http://www.pactomundial.org.mx/default800.htm(C 